\title{
Ischemia reperfusion-facilitated sinusoidal endothelial cell injury in liver transplantation and the resulting impact of extravasated platelet aggregation
}

\author{
T. Miyashita - S. Nakanuma - A. K. Ahmed - I. Makino - H. Hayashi · \\ K. Oyama - H. Nakagawara $\cdot$ H. Tajima $\cdot$ H. Takamura $\cdot$ I. Ninomiya $\cdot$ \\ S. Fushida $\cdot$ J. W. Harmon · T. Ohta
}

Received: 8 July 2015 / Revised: 28 September 2015 / Accepted: 29 September 2015 / Published online: 14 October 2015 (C) Springer-Verlag Wien 2015

\section{Summary}

Background The exact sequence of events leading to ultimate hepatocellular damage following ischemia/ reperfusion $(\mathrm{I} / \mathrm{R})$ is incompletely understood. In this article, we review a mechanism of organ dysfunction after hepatic I/R or immunosuppressive treatment, in addition to the potential of liver sinusoidal endothelial cell (LSEC) protection and antiplatelet treatment for the suppression of hepatocellular damage.

Methods A review of the literature, utilizing PubMedNCBI, was used to provide information on the components necessary for the development of hepatocellular damage following I/R.

Results It is well-established that LSECs damage following hepatic I/R or immunosuppressive treatment followed by extravasated platelet aggregation (EPA) is the

T. Miyashita, MD, $\mathrm{PhD}(\bowtie) \cdot \mathrm{S}$. Nakanuma, MD, $\mathrm{PhD}$.

I. Makino, MD, PhD $\cdot$ H. Hayashi, MD, PhD $\cdot$ K. Oyama, MD,

$\mathrm{PhD} \cdot \mathrm{H}$. Nakagawara, MD, PhD $\cdot \mathrm{H}$. Tajima, MD, PhD

H. Takamura, MD, PhD $\cdot$ I. Ninomiya, MD, PhD · S. Fushida, MD, $\mathrm{PhD} \cdot \mathrm{T}$. Ohta, MD, PhD

Department of Gastroenterological Surgery, Kanazawa University Hospital,

13-1 Takaramachi,

920-8641 Kanazawa, Ishikawa, Japan

e-mail: tomoharumiya@gmail.com

S. Nakanuma, MD, PhD

e-mail: n_shin@gj8.so-net.ne.jp

I. Makino, $\mathrm{MD}, \mathrm{PhD}$

e-mail: i.makino@staff.kanazawa-u.ac.jp

H. Hayashi, MD, PhD

e-mail: ekhayashi@hotmail.com

K. Oyama, MD, PhD

e-mail: oya-ma@staff.kanazawa-u.ac.jp

H. Nakagawara, MD, PhD

e-mail: nakagawarahisatoshi@gmail.com root cause of organ dysfunction in liver transplantation. We have classified three phases, from LSECs damage to organ dysfunction, utilizing the predicted pathogenic mechanism of sinusoidal obstruction syndrome. The first phase is detachment of LSECs and sinusoidal wall destruction after LSECs injury by hepatic I/R or immunosuppressive treatment. The second phase is EPA, accomplished by sinusoidal wall destruction. The various growth factors, including thromboxane A2, serotonin, transforming growth factor-beta and plasminogen activator inhibitor-1, released by EPA in the Disse's space of zone three, induce portal hypertension and the progression of hepatic fibrosis. The third phase is organ dysfunction following portal hypertension, hepatic fibrosis, and suppressed liver regeneration through various growth factors secreted by EPA.

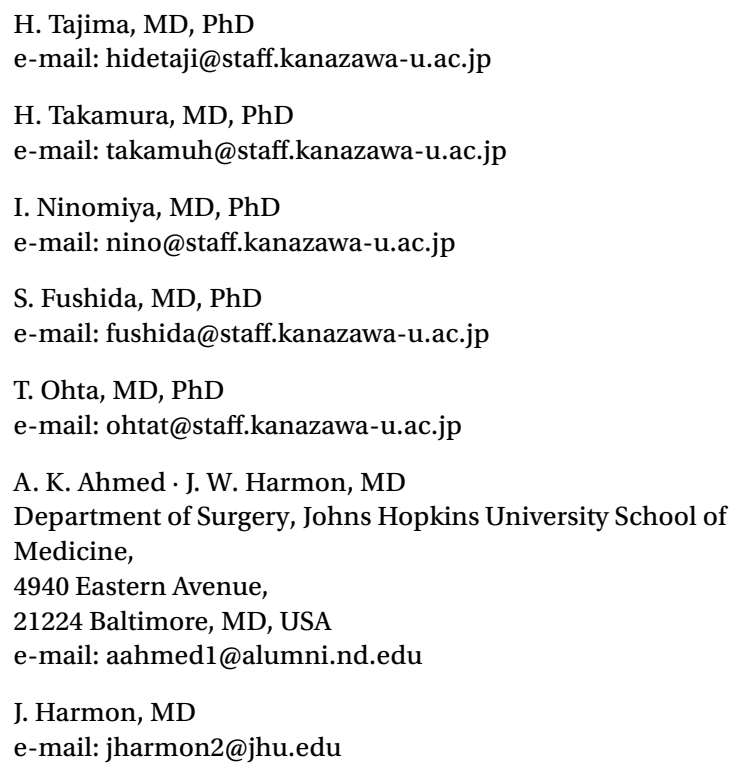


Conclusion We suggest that EPA in the space of Disse, initiated by LSECs damage due to hepatic I/R or immunosuppressive treatment, and activated platelets may primarily contribute to liver damage in liver transplantation. Endothelial protective therapy or antiplatelet treatment may be useful in the treatment of hepatic I/R following EPA.

Keywords Ischemia/reperfusion - Extravasated platelet aggregation · Sinusoidal endothelial damage · Antiplatelet agents $\cdot$ Endothelial protection

\section{Introduction}

Ischemia/reperfusion (I/R) injury to the liver is a major complication of hemorrhagic shock, liver resection, and transplantation [1]. Although the sequence of events that leads to extent of hepatocellular damage after hepatic I/R is incompletely understood, liver sinusoidal endothelial cells (LSECs) may loosen from their tetherings to the space of Disse in zone 3 or even detach completely [2].

Hepatic I/R is considered as a biphasic phenomenon. Cellular damage due to hypoxia and a lack of biomechanical stimulus is exacerbated upon the restoration of oxygen delivery and shear stress [3]. Although the initial ischemic insult to the liver is tolerable, this first step triggers essential molecules in the induction of the more devastating reperfusion injury. Early phases of reperfusion are characterized pathologically by endothelial cell swelling, vasoconstriction, neutrophil entrapment, and platelet aggregation within the sinusoids-resulting in failure of the microcirculation [4]. Simultaneously, nitric oxide (NO) levels are markedly reduced and there is an imbalance between endothelin-1 and NO production from NO synthase (NOS). This leads to vasoconstriction of the sinusoids [5]. As such, vasoconstriction of the sinusoids gives rise to narrowing of the sinusoidal lumen with consequential decreased leukocyte velocity. The frequency of leukocyte-endothelial cell contact is elevated, promoting leukostasis. Flow is hindered in the sinusoidal network of the hepatic microcirculation due to stagnant leukocytes, unable to completely occlude the sinusoidal lumen [6]. Microcirculatory failure leads to aggravated and prolonged ischemia. Hypoxic regions of the liver heighten the degree of necrosis, Kupffer cell activation, and induce further cytokine and reactive oxygen species release. This creates a cycle of excessive inflammatory response, reactive oxygen and nitrogen species production, and further oxidative tissue injury [7].

LSECs, which lack an organized basal membrane, form the vascular wall of the hepatic sinusoid. Fenestrations penetrate the cytoplasm of these flattened cells forming clusters, called sieve plates, which render the hepatic microvascular endothelium discontinuous [8]. LSECs play an integral protective role in maintaining vascular homeostasis, inflammation, vascular tone, and toxicant clearance. Thus, the preservation of a healthy LSEC phenotype is fundamental to minimize any type of liver injury [3]. Damage to the endothelium, following hepatic I/R injury, is apparent within LSECs and hepatocytes, indicated by the deposition of fibrinogen and erythrocyte congestion resulting in enlarged sinusoids. Sloughed LSECs, red cells, and stellate cells embolize downstream, leading to venous occlusions that progress to disrupt the normal liver architecture and achieve centrilobular necrosis [9]. In the late phases of the disease, fibrosis and occlusion of the terminal venules develop, leading to hepatic failure and possibly death [10].

Sinusoidal obstruction syndrome (SOS), previously known as veno-occlusive disease, commences with sustained LSECs injury, resulting in bleeding in the space of Disse and centrilobular hemorrhagic necrosis. The fundamental cause, moreover, is damage around the centrilobular area, including the sinusoid, by acute cellular rejection, antibody-mediated rejection or hepatic I/R injury [11]. SOS is a life-threatening syndrome that results from sinusoidal congestion and is characterized by hepatomegaly, ascites, portal hypertension, weight gain and jaundice [12].

Platelets play an important role in hepatocellular damage. Furthermore, platelets have been suggested to be involved in the inflammatory response of hepatic I/R injury in various organs [13]. They are able to roll and adhere to postreperfusion endothelium in a P-selectindependent manner $[14,15]$. Platelets accumulate in the postischemic microvasculature early after reperfusion via P-selectin-ligand interactions. Platelet recruitment and subsequent activation might play an important role in the pathogenesis of hepatic I/R injury [14]. Platelet aggregation also correlated with reperfusion injury, thrombocytopenia and early graft dysfunction in liver transplantation [16]. As such, persistent thrombocytopenia after reperfusion is an unfavorable indicator for early liver graft dysfunction [17].

We previously reported that platelet aggregation in the space of Disse along with the sinusoid and platelets phagocytosis by hepatocytes were observed in the allograft tissue of a living donor liver transplantation recipient with thrombocytopenia, who encountered a complication of SOS [18]. Therefore, LSECs damage after hepatic I/R or immunosuppressive treatment followed by extravasated platelet aggregation (EPA) is the root cause of organ dysfunction in liver transplantation.

This manuscript will review the role of platelet aggregation and possible prevention for hepatocyte injury in hepatic I/R. The PubMed database was utilized in searching for published literature in this area.

\section{Platelets and hepatic ischemia/reperfusion injury}

Whereas many of the mechanisms underlying the hepatic I/R-induced inflammatory response still remain unknown, growing evidence suggests a role for platelets in the pathogenesis of postischemia reperfusion injuryw [14]. Ischemia leads to the accumulation and activation 


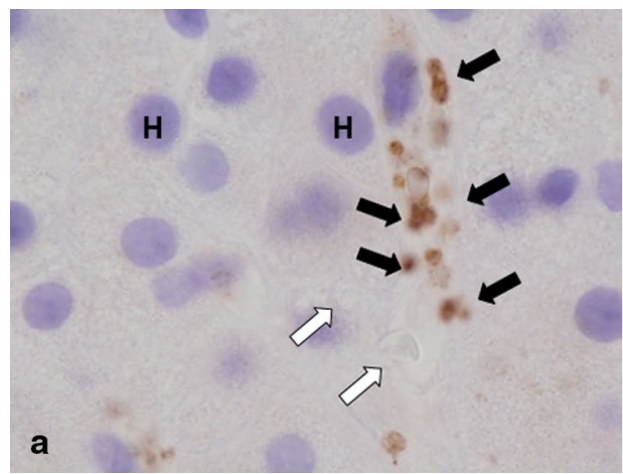

Fig. 1 Immunohistochemical staining of liver allograft tissues with antibody to CD42b. a. CD42b expression is evident as dark particles, morphologically characterized as platelets (black arrows). Platelets were observed in the sinusoid in zone 3.

of platelets within vascular beds early after reperfusion [19].

We reported that $\mathrm{CD} 42 \mathrm{~b}$ expression, as a platelet marker, was apparent along with the sinusoid in Zone 3 but not in Zone 1 in liver allograft tissue (Fig. 1a).

Remarkably, superior adenosine diphosphate (ADP)dependent aggregative levels of platelets prior to graft reperfusion were demonstrated to be positively correlated with serum markers for hepatocellular reperfusion damage. Schulte et al. reported that a certain increase in the ADP-dependent functional status of platelets in the pediatric recipients of liver grafts may result in a critical promotion of platelet adherence and activation in liver graft LSECs following reperfusion [16, 20]. Platelet accumulation in the postischemic microvasculature might significantly contribute to the manifestation of hepatic I/R injury [14].

Extracellular nucleotides are released in a regulated manner either by platelets, a variety of vascular and hepatic cells in response to inflammatory stress, through cellular swelling, or with exocytosis. Levels of extracellular nucleotides are, in turn, regulated by CD39 (ectonucleoside triphosphate diphosphohydrolase-1/ ENTPD1), the dominant vascular ectonucleotidase. CD39, expressed only on the luminal surface of proliferating or activated LSECs and absent in hepatocytes [21, 22], hydrolyzes the terminal phosphate of adenosine triphosphate(ATP) and ADP in an enzymatic cascade that generates adenosine monophosphate(AMP) [23]. Under normal conditions, in the absence of stressors such as hypoxia/ischemia, high shear stress, and triggers for inflammation (cytokines/chemokines), CD39 helps to maintain a homeostatic vascular environment, maintain blood fluidity and inhibit inflammation [24]. ADP, one of the most potent signals for platelet aggregation, as well as proinflammatory signals such as ATP, are released into the extracellular environment in the presence of adverse conditions, such as hepatic I/R. When this occurs, the extracellular concentration of ADP and ATP increases markedly [25]. When platelets contact collagen, they produce ADP, which further promotes platelet aggregation.

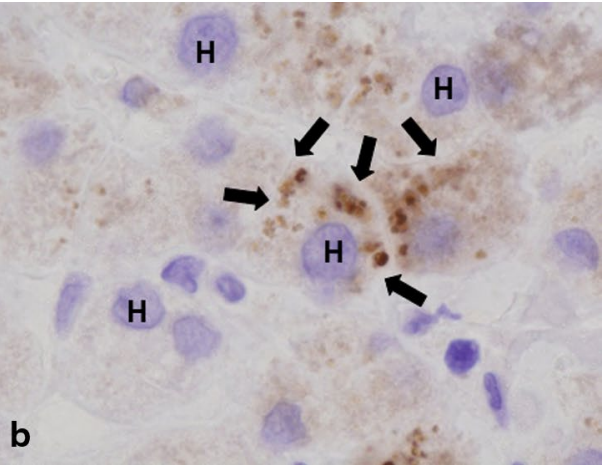

Erythrocytes were evident in the sinusoid (white arrows). b CD42b expression was observed in extravasation or in the hepatocyte cytoplasm (black arrows), indicative of extravasated platelet aggregation. Hepatocytes are denoted by " $H$ ".

Platelets release potent proinflammatory chemokines and modulate leukocyte function [26]. Activated platelets release growth factors, such as thromboxane (TX) A2, serotonin, vascular endothelial growth factor (VEGF)-A, transforming growth factor (TGF)- $\beta$ and plasminogen activator inhibitor (PAI)-1. TXA2 is a strong vasoactive metabolite of arachidonic acid with powerful proaggregatory and proinflammatory properties. TXA2 is a vasoconstrictor that increases portal venous resistance [27] and causes portal hypertension. Serotonin is well-known released from platelets on damage to the blood vessels walls. It acts as a potent vasoconstrictor. Although VEGFA acts as a vasodilator under ordinary circumstances, it acts, paradoxically, as a vasoconstrictor in patients with endothelial failure [28]. Bevacizumab, an antibody against VEGF-A, protects against liver injury associated with SOS [29]. PAI-1 suppresses fibrinolysis and the progression to fibrosis in the tissue microenvironment. In addition, PAI- 1 acts as a negative regulator of hepatocyte proliferation by inhibiting urokinase-type plasminogen activator (u-PA), which activates hepatocyte growth factor $[30,31]$. TGF- $\beta$, a major antiproliferative factor for hepatocytes, stimulates collagen synthesis through activated hepatic stellate cells (HSCs) [32].

LSEC apoptosis also play an important role in hepatic I/R injury during liver transplantation [33]. Platelet sequestration occurs after transplantation with possibly deleterious effects. In an isolated perfused rat liver model, single platelets were adherent to sinusoidal lining without morphological or dynamic evidence of impairment in microcirculation with increase in the number of apoptotic LSECs. Platelets cause LSECs apoptosis upon reperfusion of liver grafts. These results indicate that the prevention of adhesion plays a protective role [15].

\section{Extravasated platelet aggregation and organ dysfunction}

The liver can be subjected to three forms of ischemia, namely cold (or hypothermic), warm (or normothermic), 
and rewarming [34]. In transplanted liver ischemia, disruption of the endothelial wall leads to leukocyte [35] and platelet adhesion $[15,36]$, which induces microcirculatory disturbances [37]. Transplanted liver damage is slightly different from liver resection, due to the duration of sustained ischemia.

The subendothelial space of Disse, located between the hepatocytes and the sinusoid, contains HSCs (myofibroblasts) and a network of reticular fibers holding the hepatocytes together. Large amounts of albumin and other plasma proteins enter the space of Disse, pass through hepatocyte junctions, and form ascites containing a high concentration of protein and a low serum to ascites albumin gradient. In normal liver, the space of Disse contains a matrix of basement-membrane constituents that is not electron dense [38]; in injured tissue this may be replaced by matrix filled with fibril-forming collagens and fibronectin [39].

In the normal liver, collagen type III are concentrated in the portal tracts and around terminal hepatic veins, with occasional bundles located between hepatocytes and endothelial cells in the space of Disse. Hepatic ischemia causes endothelial cell activation following HSCs activation, increasing hepatic parenchymal and portal tract fibrosis in ischemic liver tissues [40]. Following a fibrogenic stimulus, HSCs undergo a complex process of activation in which they become transformed from quiescent to activated myofibroblast-like cells [41-43]. Activated HSCs are the primary cell type responsible for the production of collagen I. This subendothelial accumulation of collagen, termed "capillarization" of the sinusoid, is associated with clinical liver disease [44]. Fibrosis may also impede the rapid exchange of solutes between the sinusoidal space and hepatocytes [45].

Following the destruction of endothelial wall and resulting LSECs damage caused by hepatic I/R or immunosuppressive treatment, platelets enter the space of Disse (Fig. 1b) and aggregate by activated HSCs (Fig. 2). Furthermore, platelets produce platelet-derived growth factor TGF- $\beta$ to activate HSCs and promote fibrosis [46]. Therefore, liver fibrosis may be caused by EPA in this pathway (Fig. 3).

The production of collagen I may be associated with increased serum alanine transaminase level and be utilized as a marker for hepatic I/R injury.

\section{Possible treatment}

No standard method for treating hepatic dysfunction by hepatic I/R has yet been established. Systemic anticoagulation and thrombolytic therapies have been tested extensively [47]. Defibrotide, a polydeoxyribonucleic acid, was recently shown to have a promising response rate in patients with severe SOS [48]. Conditional to the extent of LSECs damage and EPA in the space of Disse, prophylactic administration of endothelial protective and antiplatelet agents may be effective prior to the development of irreversible damage.

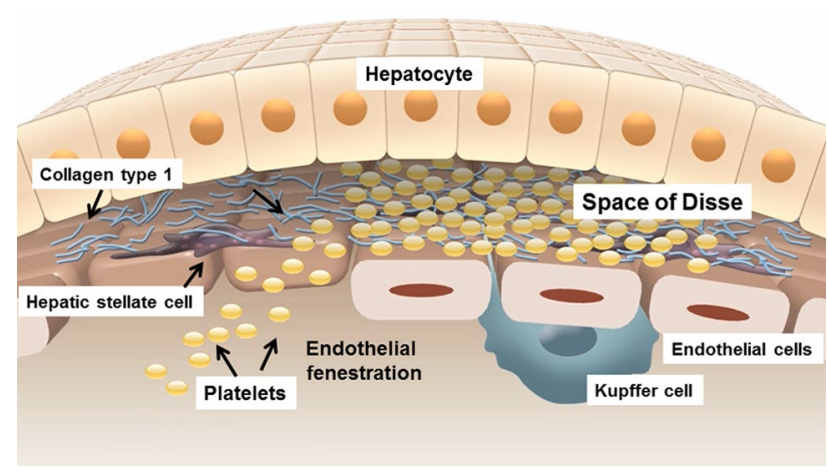

Fig. 2 Extravasated platelet aggregation schematic mechanism. Damage to the sinusoidal endothelium can result in the denuding of the endothelium or the loss of fenestrations, allowing platelets to enter the space of Disse. This space contains reticulin fibers, consisting primarily of collagen type III. Platelets can easily attach to collagen type III, forming aggregates in the space of Disse. Activated hepatic stellate cell synthesize a fibrotic matrix rich in collagen type I. In addition, the extravasated platelets in the space of Disse can be phagocytized by hepatocytes through the asialoglycoprotein receptor.

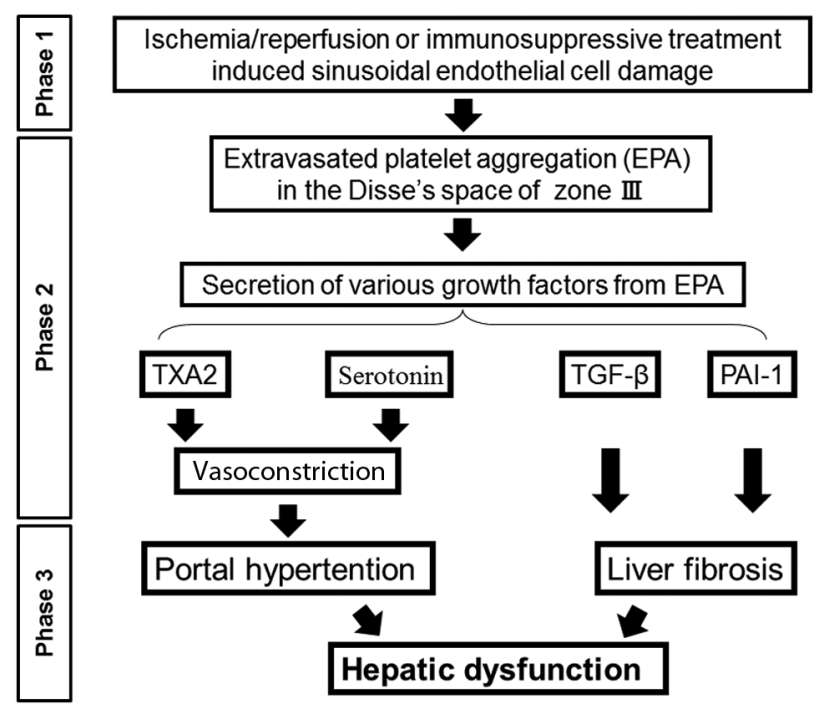

Fig. 3 Mechanisms involved in hepatic injury after liver ischemia/reperfusion. EPA in the space of Disse, initiated by damage to the sinusoidal endothelium, is induced by ischemia/reperfusion or immunosuppressive treatment. The various growth factors released by activated platelets, including thromboxane $\mathrm{A} 2$, serotonin, plasminogen activator inhibitor-1, and transforming growth factor- $\beta$, may induce portal hypertension and the progression of hepatic fibrosis, as well as suppression of liver regeneration, initiating hepatic dysfunction

Additionally, antiapoptosis induced by ishchemic preconditioning can improve liver function, as well as protect LSECs [49]. Ischemic preconditioning directly protected hepatocytes after warm I/R, not via suppression of alterations in sinusoidal cells, as is present in cold hepatic I/R injury [50]. 


\section{Reinforcement and protection of LSECs}

Hyperglycemia, indicative of diabetes mellitus, is a major risk factor for endothelial dysfunction and vascular complications. In recent years, significant advances have been made in understanding endothelial cell dysfunction, triggered by high glucose concentration [51]. In order to prevent graft injury during perioperatively tight glycemic control, it is necessary to reduce the risk of microangiopathic organ injury by preventing endothelial cell injury [52]. The artificial pancreas might have beneficial effects in liver transplant recipients due to the hepatoprotective effects of insulin, including the stimulation of regeneration and endothelial cell protection [53]. Additionally, insulin has an antiinflammatory effect through the suppression of inflammatory cytokines (e.g, nuclear factor kappa B) [54]. To ascertain this, we applied a closed-loop glycemic control system with an artificial pancreas (STG-55, NIKKISO CO., LTD., Tokyo, Japan) to the liver transplant recipient.

In obese patients with type 2 diabetes, insulin delivery to and insulin-dependent glucose uptake by skeletal muscle are delayed and impaired. Therefore, it is pivotal to impair insulin resistance for endothelial cells. Kubota et al. [55] demonstrated that impaired insulin signaling in endothelial cells, due to reduced insulin receptor substrate two expression and insulin-induced eNOS phosphorylation, causes attenuation of insulin-induced capillary recruitment and insulin delivery, reducing glucose uptake by skeletal muscle. The use of agents such as Beraprost, a stable prostacyclin analogue (PGI2), capable of improving insulin resistance and resulting vascular endothelial function, may ultimately contribute to increasing the life expectancy of patients with peripheral artery disease [56]. To improve insulin resistance, we administer Beraprost sodium during the perioperative state.

\section{Antiplatelet agents therapy}

Phosphodiesterase 3 (PDE3) inhibitors Cilostazol and Milrinone may be appropriate, owing to their antiplatelet properties, ability to increase tolerance to hepatic I/R injury [57], and induction of immune tolerance via an enhanced regulatory T-cell response [58]. Furthermore, PDE3 inhibition regulates endothelial CD39 at a posttranslational level [25]. To reaffirm such a treatment, a few experimental studies in rat models demonstrated that PDE3 inhibitors protected SOS [59], and attenuated graft injury using an orthotopic liver transplant model [60].

In clinical practice, we administered a PDE3 inhibitor with the use of local infusion therapy to living donor liver transplant recipients or patients with small remnant liver volume after a hepatectomy such as in tri-segmentectomy, with favorable outcomes [18].

Beraprost also prevents platelet aggregation by increasing cyclic AMP to effectively reduce the amount of TXA2, which has coagulant properties and is made by platelets. Beraprost is also administered as an antiplatelet agent.

\section{Conclusion}

We suggest that EPA in the space of Disse, initiated by LSECs damage due to hepatic I/R or immunosuppressive treatment, and activated platelets may primarily contribute to liver damage in liver transplantation. Endothelial protective therapy or antiplatelet treatment may be useful in the treatment of hepatic I/R following EPA.

\section{Conflict of interest}

The authors declares that there is no conflict of interests regarding the publication of this paper.

\section{Funding}

The authors received no financial support for the research, author-ship, and/or publication of this article.

\section{References}

1. Clarke CN, Kuboki S, Tevar A, Lentsch AB, Edwards M. Cxc chemokines play a critical role in liver injury, recovery, and regeneration. Am J Surg. 2009;198:415-9.

2. DeLeve LD. Hepatic microvasculature in liver injury. Semin Liver Dis. 2007;27:390-400.

3. Peralta C, Jiménez-Castro MB, Gracia-Sancho J. Hepatic ischemia and reperfusion injury: effects on the liver sinusoidal milieu. J Hepatol. 2013;59:1094-106.

4. Serracino-Inglott F, Habib NA, Mathie RT. Hepatic ischemia-reperfusion injury. Am J Surg. 2001;181:160-6.

5. Marzi I, Takei Y, Rücker M, Kawano S, Fusamoto H, Walcher F, Kamada T. Endothelin-1 is involved in hepatic sinusoidal vasoconstriction after ischemia and reperfusion. Transpl Int. 1994;7(Suppl 1):S503-S6.

6. Vollmar B, Richter S, Menger MD. Leukocyte stasis in hepatic sinusoids. Am J Physiol. 1996;270:G798-803.

7. Nastos C, Kalimeris K, Papoutsidakis N, Tasoulis MK, Lykoudis PM, Theodoraki K, Nastou D, Smyrniotis V, Arkadopoulos N. Global consequences of liver ischemia/reperfusion injury. Oxid Med Cell Longev. 2014;2014:906965.

8. Wisse E. An ultrastructural characterization of the endothelial cell in the rat liver sinusoid under normal and various experimental conditions, as a contribution to the distinction between endothelial and kupffer cells. J Ultrastruct Res. 1972;38:528-62.

9. Shulman HM, Fisher LB, Schoch HG, Henne KW, McDonald GB. Veno-occlusive disease of the liver after marrow transplantation: histological correlates of clinical signs and symptoms. Hepatology. 1994;19:1171-81.

10. Chao N. How I treat sinusoidal obstruction syndrome. Blood. 2014;123:4023-6.

11. Takamura H, Nakanuma S, Hayashi H, Tajima H, Kakinoki K, Kitahara M, Sakai S, Makino I, Nakagawara H, Miyashita T, Okamoto K, Nakamura K, Oyama K, Inokuchi M, Ninomiya I, Kitagawa H, Fushida S, Fujimura T, Onishi I, Kayahara M, Tani T, Arai K, Yamashita T, Kitamura H, Ikeda H, Kaneko S, Nakanuma Y, Matsui O, Ohta T. Severe venoocclusive disease/sinusoidal obstruction syndrome after deceased-donor and living-donor liver transplantation. Transplant Proc. 2014;46:3523-35. 
12. Campos-Varela I, Castells L, Dopazo C, Pérez-Lafuente M, Allende H, Len O, Llopart L, Vargas V, Charco R. Transjugular intrahepatic portosystemic shunt for the treatment of sinusoidal obstruction syndrome in a liver transplant recipient and review of the literature. Liver Transpl. 2012;18:201-5.

13. de Vries DK, Schaapherder AF, Reinders ME. Mesenchymal stromal cells in renal ischemia/reperfusion injury. Front Immunol. 2012;3:162.

14. Massberg S, Enders G, Leiderer R, Eisenmenger S, Vestweber D, Krombach F, Messmer K. Platelet-endothelial cell interactions during ischemia/reperfusion: the role of P-selectin. Blood. 1998;92:507-15.

15. Sindram D, Porte RJ, Hoffman MR, Bentley RC, Clavien PA. Platelets induce sinusoidal endothelial cell apoptosis upon reperfusion of the cold ischemic rat liver. Gastroenterology. 2000;118:183-91.

16. Schulte am Esch J, Akyildiz A, Tustas RY, Ganschow R, Schmelzle M, Krieg A, Robson SC, Topp SA, Rogiers X, Knoefel WT, Fischer L. Adp-dependent platelet function prior to and in the early course of pediatric liver transplantation and persisting thrombocytopenia are positively correlated with ischemia/reperfusion injury. Transpl Int. 2010;23:745-52.

17. McCaughan GW, Herkes R, Powers B, Rickard K, Gallagher ND, Thompson JF, Sheil AG. Thrombocytopenia post liver transplantation. Correlations with pre-operative platelet count, blood transfusion requirements, allograft function and outcome. J Hepatol. 1992;16:16-22.

18. Nakanuma S, Miyashita T, Hayashi H, Tajima H, Takamura H, Tsukada T, Okamoto K, Sakai S, Makino I, Kinoshita J, Nakamura K, Oyama K, Inokuchi M, Nakagawara H, Ninomiya I, Kitagawa H, Fushida S, Fujimura T, Ohta T. Extravasated platelet aggregation in liver zone 3 may correlate with the progression of sinusoidal obstruction syndrome following living donor liver transplantation: a case report. Exp Ther Med. 2015;9:1119-24.

19. Chintala MS, Bernardino V, Chiu PJ. Cyclic gmp but not cyclic amp prevents renal platelet accumulation after ischemia-reperfusion in anesthetized rats. J Pharmacol Exp Ther. 1994;271:1203-8.

20. Plevak DJ, Halma GA, Forstrom LA, Dewanjee MK, O'Connor MK, Moore SB, Krom RA, Rettke SR. Thrombocytopenia after liver transplantation. Transplant Proc. 1988;20:630-3.

21. Enjyoji K, Sévigny J, Lin Y, Frenette PS, Christie PD, Esch JS, Imai M, Edelberg JM, Rayburn H, Lech M, Beeler DL, Csizmadia E, Wagner DD, Robson SC, Rosenberg RD. Targeted disruption of cd39/ATP diphosphohydrolase results in disordered hemostasis and thromboregulation. Nat Med. 1999;5:1010-7.

22. Goepfert C, Sundberg C, Sévigny J, Enjyoji K, Hoshi $\mathrm{T}$, Csizmadia E, Robson S. Disordered cellular migration and angiogenesis in cd39-null mice. Circulation. 2001;104:3109-15.

23. Koziak K, Sévigny J, Robson SC, Siegel JB, Kaczmarek E. Analysis of CD39/ATP diphosphohydrolase (ATPDase) expression in endothelial cells, platelets and leukocytes. Thromb Haemost. 1999;82:1538-44.

24. Eltzschig HK, Köhler D, Eckle T, Kong T, Robson SC, Colgan SP. Central role of Sp1-regulated CD39 in hypoxia/ischemia protection. Blood. 2009;113:224-32.

25. Baek AE, Kanthi Y, Sutton NR, Liao H, Pinsky DJ. Regulation of ecto-apyrase cd39 (ENTPD1) expression by phosphodiesterase III (PDE3). FASEB J. 2013;27:4419-28.
26. Ghasemzadeh M, Hosseini E. Platelet-leukocyte crosstalk: linking proinflammatory responses to procoagulant state. Thromb Res. 2013;131:191-7.

27. Ruan Z, Shibamoto T, Shimo T, Koizumi T, Tsuchida H, Kurata Y, Ogura T, Kubo K. Effects of platelet-activating factor and thromboxane A2 on isolated perfused guinea pig liver. Prostaglandins Other Lipid Mediat. 2004;73:73-85.

28. Parenti A, Brogelli L, Filippi S, Donnini S, Ledda F. Effect of hypoxia and endothelial loss on vascular smooth muscle cell responsiveness to VEGF-A: role of flt-1/VEGF-receptor-1. Cardiovasc Res. 2002;55:201-12.

29. Ribero D, Wang H, Donadon M, Zorzi D, Thomas MB, Eng C, Chang DZ, Curley SA, Abdalla EK, Ellis LM, Vauthey JN. Bevacizumab improves pathologic response and protects against hepatic injury in patients treated with oxaliplatinbased chemotherapy for colorectal liver metastases. Cancer. 2007;110:2761-7.

30. Mars WM, Zarnegar R, Michalopoulos GK. Activation of hepatocyte growth factor by the plasminogen activators uPA and tPA. Am J Pathol. 1993;143:949-58.

31. Watanabe K, Togo S, Takahashi T, Matsuyama R, Yamamoto H, Shimizu T, Makino H, Matsuo K, Morioka D, Kubota T, Nagashima Y, Shimada H. PAI-1 plays an important role in liver failure after excessive hepatectomy in the rat. J Surg Res. 2007;143:13-9.

32. Ueda S, Yamanoi A, Hishikawa Y, Dhar DK, Tachibana M, Nagasue N. Transforming growth factor-betal released from the spleen exerts a growth inhibitory effect on liver regeneration in rats. Lab Invest. 2003;83:1595-603.

33. Cursio R, Miele C, Filippa N, Van Obberghen E, Gugenheim J. Liver HIF-1 alpha induction precedes apoptosis following normothermic ischemia-reperfusion in rats. Transplant Proc. 2008;40:2042-5.

34. Selzner N, Rudiger H, Graf R, Clavien PA. Protective strategies against ischemic injury of the liver. Gastroenterology. 2003;125:917-36.

35. Takei Y, Marzi I, Gao WS, Gores GJ, Lemasters JJ, Thurman RG. Leukocyte adhesion and cell death following orthotopic liver transplantation in the rat. Transplantation. 1991;51:959-65.

36. Cywes R, Packham MA, Tietze L, Sanabria JR, Harvey PR, Phillips MJ, Strasberg SM. Role of platelets in hepatic allograft preservation injury in the rat. Hepatology. 1993;18:635-47.

37. Marzi I, Knee J, Menger MD, Harbauer G, Bühren V. Hepatic microcirculatory disturbances due to portal vein clamping in the orthotopic rat liver transplantation model. Transplantation. 1991;52:432-6.

38. Bissell DM, Arenson DM, Maher JJ, Roll FJ. Support of cultured hepatocytes by a laminin-rich gel. Evidence for a functionally significant subendothelial matrix in normal rat liver. J Clin Invest. 1987;79:801-12.

39. Martinez-Hernandez A. The hepatic extracellular matrix. II. Electron immunohistochemical studies in rats with CCl4-induced cirrhosis. Lab Invest. 1985;53:166-86.

40. Cheng F, Li Y, Feng L, Li S. Hepatic stellate cell activation and hepatic fibrosis induced by ischemia/reperfusion injury. Transplant Proc. 2008;40:2167-70.

41. Friedman SL. Molecular regulation of hepatic fibrosis, an integrated cellular response to tissue injury. J Biol Chem. 2000;275:2247-50.

42. Lieber CS. Metabolism of alcohol. Clin Liver Dis. 2005;9:1-35.

43. Vera M, Nieto N. Hepatic stellate cells and alcoholic liver disease. Rev Esp Enferm Dig. 2006;98:674-84.

44. Schaffner F, Poper H. Capillarization of hepatic sinusoids in man. Gastroenterology. 1963;44:239-42. 
45. Friedman SL. Seminars in medicine of the Beth Israel Hospital, Boston. The cellular basis of hepatic fibrosis. Mechanisms and treatment strategies. N Engl J Med. 1993;328:1828-35.

46. Yoshida S, Ikenaga N, Liu SB, Peng ZW, Chung J, Sverdlov DY, Miyamoto M, Kim YO, Ogawa S, Arch RH, Schuppan D, Popov Y. Extrahepatic platelet-derived growth factor- $\beta$, delivered by platelets, promotes activation of hepatic stellate cells and biliary fibrosis in mice. Gastroenterology. 2014;147:1378-92.

47. Yamada N, Urahashi T, Ihara $\mathrm{Y}$, Sanada $\mathrm{Y}$, Wakiya $\mathrm{T}$, Okada N, Mizuta K. Veno-occlusive disease/sinusoidal obstruction syndrome associated with potential antibodymediated rejection after pediatric living donor liver transplantation: a case report. Transplant Proc. 2012;44:810-3.

48. Zhang L, Wang Y, Huang H. Defibrotide for the prevention of hepatic veno-occlusive disease after hematopoietic stem cell transplantation: a systematic review. Clin Transplant. 2012;26:511-9.

49. Sindram D, Rüdiger HA, Upadhya AG, Strasberg SM, Clavien $P A$. Ischemic preconditioning protects against cold ischemic injury through an oxidative stress dependent mechanism. J Hepatol. 2002;36:78-84.

50. Tejima K, Arai M, Ikeda H, Tomiya T, Yanase M, Inoue $Y$, Nagashima K, Nishikawa T, Watanabe N, Omata M, Fujiwara $\mathrm{K}$. Ischemic preconditioning protects hepatocytes via reactive oxygen species derived from kupffer cells in rats. Gastroenterology. 2004;127:1488-96.

51. Georgescu A, Popov D, Dragan E, Dragomir E, Badila E. Protective effects of nebivolol and reversal of endothelial dysfunction in diabetes associated with hypertension. Eur J Pharmacol. 2007;570:149-58.

52. Hanazaki K, Kitagawa H, Yatabe T, Munekage M, Dabanaka K, Takezaki Y, Tsukamoto Y, Asano T, Kinoshita Y, Namikawa T. Perioperative intensive insulin therapy using an artificial endocrine pancreas with closed-loop glycemic control system: the effects of no hypoglycemia. Am J Surg. 2014;207:935-41.
53. Hayashi H, Takamura H, Nakanuma S, Makino I, Tajima H, Fushida S, Hanazaki K, Ohta T. Application of an artificial pancreas for a liver transplant recipient. Exp Clin Transplant. 2014;12:572-3.

54. Wang S, Moustaid-Moussa N, Chen L, Mo H, Shastri A, Su R, Bapat P, Kwun I, Shen CL. Novel insights of dietary polyphenols and obesity. J Nutr Biochem. 2014;25:1-18.

55. Kubota $T$, Kubota $N$, Kumagai H, Yamaguchi S, Kozono $\mathrm{H}$, Takahashi T, Inoue M, Itoh S, Takamoto I, Sasako T, Kumagai K, Kawai T, Hashimoto S, Kobayashi T, Sato M, Tokuyama K, Nishimura S, Tsunoda M, Ide T, Murakami K, Yamazaki T, Ezaki O, Kawamura K, Masuda H, Moroi M, Sugi K, Oike Y, Shimokawa H, Yanagihara N, Tsutsui M, Terauchi Y, Tobe K, Nagai R, Kamata K, Inoue K, Kodama T, Ueki K, Kadowaki T. Impaired insulin signaling in endothelial cells reduces insulin-induced glucose uptake by skeletal muscle. Cell Metab. 2011;13:294-307.

56. Utsunomiya K. Treatment strategy for type 2 diabetes from the perspective of systemic vascular protection and insulin resistance. Vasc Health Risk Manag. 2012;8:429-36.

57. Iba T, Kidokoro A, Fukunaga M, Takuhiro K, Ouchi M, Ito Y. Comparison of the protective effects of type III phosphodiesterase (PDE3) inhibitor (cilostazol) and acetylsalicylic acid on intestinal microcirculation after ischemia reperfusion injury in mice. Shock. 2006;26:522-6.

58. Wang S, Yan C, Xu H, Zhao X, Han Y. Suppression of encephalitogenic T-cell responses by cilostazol is associated with upregulation of regulatory $\mathrm{T}$ cells. Neuroreport. 2010;21:629-35.

59. Narita M, Hatano E, Ikai I, Miyagawa-Hayashino A, Yanagida A, Nagata H, Asechi H, Taura K, Uemoto S. A phosphodiesterase III inhibitor protects rat liver from sinusoidal obstruction syndrome through heme oxygenase-1 induction. Ann Surg. 2009;249:806-13.

60. Ikegami T, Nishizaki T, Hiroshige S, Ohta R, Yanaga K, Sugimachi K. Experimental study of a type 3 phosphodiesterase inhibitor on liver graft function. Br J Surg. 2001;88:59-64. 